\title{
Digital heart: personalized medicine and inverse problems
}

\author{
A. Prikhodko ${ }^{3 *}$, M. Shishlenin ${ }^{1,2,3}$ \\ ${ }^{1}$ Sobolev Institute of Mathematics SB RAS, Novosibirsk, Russia \\ ${ }^{2}$ Institute of Computational Mathematics and Mathematical Geophysics SB RAS, Novosibirsk, Russia \\ ${ }^{3}$ Novosibirsk State University, Novosibirsk, Russia \\ *e-mail:Prikhodko1997@gmail.com,mshishlenin@ngs.ru
}

Key words: pumping function of the heart, inverse problem

Motivation and Aim: Cardiovascular disease (CVD) is the leading cause of death of the worldwide: no other cause kills as many people each year as CVD. The purpose of the study is to obtain personalized information (blood viscosity, pressure in ventricles, pulmonary artery, pulmonary vein, aorta, full vein, right atrium) about the patient by solving the inverse problem, that will help in the diagnosis of the cardiovascular system. Methods and Algorithms: We formulate the mathematical model of the heart in the form of the four-chamber pump [1]. In this pump unit, the Atria perform the function of lowpressure pumps, and the ventricles function as high-pressure pumps. The model is the system of the ordinary differential equations (ODE) and consists of the first and the second ODE.

Let us suppose that we can measure the blood flow and the pressure as the functions of time. The inverse problem consists in finding heart mass, for each patient. We reduce inverse problem to the functional minimization. Due to the extremely nonlinearity of the problem we apply the gradient method of the minimization of the cost functional.

Results: Numerical methods for solving the inverse problems are constructed.

Conclusion: The solution of inverse problem allows us to find the heart parameters for each patient (personalized medicine).

Acknowledgements: the work was supported by the MSC RK grant No. AP05134121.

\section{References}

1. Lebedenko I.S., Novoselova E.S., Rakityanskaya A.S., Efimtseva J.A. (2009). Mathematical model of the heart. Biotechnosphere. 3:24-31.

2. Lee J.-H. et al. (2003) Experimental study of sliding mode control for a benchmark magnetic bearing system and artificial heart pump suspension." IEEE Transactions on control systems technology. 11(1):128-138. 